\title{
Substratum Interpretation of the Quark-Lepton Symmetries in the Planck Aether Model of a Unified Field Theory
}

\author{
F. Winterberg \\ Desert Research Institute, University of Nevada System, Reno, Nevada 89506
}

Z. Naturforsch. 46a, 551 -559 (1991); received March 22, 1991

An attempt is made to explain the observed quark-lepton symmetries in the framework of the recently proposed Planck aether model of a unfied field theory. If the many body lepton wave function can rearrange itself into a vortex-substructure, very much as it has been observed in the fractional quantum Hall effect, all the symmetries can be understood by the quantum numbers of these vortices and their interactions, and all fermions as permitted combinations of those vortices, including the correct number of force-transmitting bosons. The proposed model can also give a value for the mass of the intermediate vector boson. All charges are explained by the quantum mechanical zero point fluctuations of the Planck masses making up the Planck aether.

\section{Introduction}

To overcome the divergence problems of relativistic quantum field theories it was recently proposed that there might be an underlying nonrelativistic superfluid substratum of densely packed positive and negative Planck masses, permeating all of space, and making up what may be called the Planck aether [1]. This hypothesis can explain fermions as objects composed of the positive and negative mass of the Planck aether, and bosons as quantized compressional and vortex waves. Lorentz invariance results as a derived dynamic symmetry for composed objects in a static equilibrium held together by forces transmitted through bosons. Symmetry demands an equal number of positive and negative Planck masses making the cosmological constant equal to zero*.

If the interactions in between the Planck masses are delta function potentials, the Planck aether obeys two fundamental equations for the two field operators $\psi_{ \pm}$

* An idea coming very close to this hypothesis was suggested by Sakharov many years ago [9]. He too believed that space might be densely filled with Planck masses and he speculated that the resulting enormous mass density would have to be compensated by hypothetical "ghost" particles. He furthermore insisted that there should be a cut-off at the Planck length, but he did not realize that the introduction of such a cut-off destroys Lorentz invariance. Accordingly, he did not take the crucial step to recognize that under these conditions, Lorentz invariance would have to be a dynamic symmetry.

Reprint requests to Prof. Dr. F. Winterberg, Desert Research Institute, University of Nevada System, Reno, Nevada 89506, USA. of the positive and negative Planck masses:

$i \hbar \frac{\partial \psi_{ \pm}}{\partial t}=\mp \frac{\hbar^{2}}{2 m_{\mathrm{p}}} \nabla^{2} \psi_{ \pm}+f^{2} r_{\mathrm{p}}^{2}\left(\psi_{+}^{\dagger} \psi_{+}-\psi_{-}^{\dagger} \psi_{-}\right) \psi_{ \pm}$

satisfying the commutation relations

$$
\begin{aligned}
& {\left[\psi_{ \pm}(\boldsymbol{r}) \psi_{ \pm}^{\dagger}\left(\boldsymbol{r}^{\prime}\right)\right]=\delta\left(\boldsymbol{r}-\boldsymbol{r}^{\prime}\right),} \\
& {\left[\psi_{ \pm}(\boldsymbol{r}) \psi_{ \pm}\left(\boldsymbol{r}^{\prime}\right)\right]=\left[\psi_{ \pm}^{\dagger}(\boldsymbol{r}) \psi_{ \pm}^{\dagger}\left(\boldsymbol{r}^{\prime}\right)\right]=0 .}
\end{aligned}
$$

In (1.1) $m_{\mathrm{p}} r_{\mathrm{p}} c=\hbar$, and $G m_{\mathrm{p}}^{2}=\hbar c$, where $m_{\mathrm{p}}$ and $r_{\mathrm{p}}$ are the Planck mass and Planck length with $G$ the Newtonian gravitational constant.

Furthermore $f^{2}=2 \hbar c$, and hence $f^{2} r_{\mathrm{p}}^{2}=2 G \hbar^{2} / c^{2}$.

Making the Hartree approximation

$$
\left\langle\psi_{ \pm}^{\dagger} \psi_{ \pm} \psi_{ \pm}\right\rangle \cong\left\langle\psi_{ \pm}^{\dagger}\right\rangle\left\langle\psi_{ \pm}\right\rangle\left\langle\psi_{ \pm}\right\rangle,
$$

whereby

$$
\varphi_{ \pm} \equiv\left\langle\psi_{ \pm}\right\rangle, \quad \varphi_{ \pm}^{*} \equiv\left\langle\psi_{ \pm}^{\dagger}\right\rangle
$$

one obtains two coupled nonlinear wave equations for $\varphi_{ \pm}$:

$$
i \hbar \frac{\partial \varphi_{ \pm}}{\partial t}=\mp \frac{\hbar^{2}}{2 m_{\mathrm{p}}} \nabla^{2} \varphi_{ \pm}+f^{2} r_{\mathrm{p}}^{2}\left(\varphi_{+}^{*} \varphi_{+}-\varphi_{-}^{*} \varphi_{-}\right) \varphi_{ \pm} .
$$

Putting

$n_{ \pm}=\varphi_{ \pm}^{*} \varphi_{ \pm}, \quad n_{ \pm} \boldsymbol{v}_{ \pm}=\mp \frac{i \hbar}{2 m_{\mathrm{p}}}\left[\varphi_{ \pm}^{*} \nabla \varphi_{ \pm}-\varphi_{ \pm} \nabla \varphi_{ \pm}^{*}\right]$

and applying the Madelung transformation [2], (1.5) assumes the hydrodynamical form

$$
\frac{\partial n_{ \pm}}{\partial t}+\operatorname{div}\left(n_{ \pm} \boldsymbol{v}_{ \pm}\right)=0, \quad \frac{\mathrm{d} \boldsymbol{v}_{ \pm}}{\mathrm{d} t}=\mp \frac{1}{m_{\mathrm{p}}} \operatorname{grad}\left(V+Q_{ \pm}\right),
$$


where

$$
\begin{aligned}
& \frac{\mathrm{d} \boldsymbol{v}_{ \pm}}{\mathrm{d} t}=\frac{\partial \boldsymbol{v}_{ \pm}}{\partial t}+\left(\boldsymbol{v}_{ \pm} \cdot \nabla\right) \boldsymbol{v}_{ \pm}, \quad V=f^{2} r_{\mathrm{p}}^{2}\left[n_{+}-n_{-}\right], \\
& Q_{ \pm}=\mp \frac{\hbar^{2}}{2 m_{\mathrm{p}}} \frac{\nabla^{2} \sqrt{n_{ \pm}}}{\sqrt{n_{ \pm}}}
\end{aligned}
$$

The Planck aether has compressional wave modes propagating with a velocity equal to the velocity of light. The source of these compressional waves are the zero point fluctuations of the Planck masses bound in the vortex filaments. The Planck masses thereby become the source of an attractive scalar force generating the Newtonian gravitational force field of the Planck masses. Without the expenditure of energy, the Planck aether can form a lattice of quantized vortex rings having a core radius equal to the Planck length $r_{\mathrm{p}}$ and a ring radius about $\sim 10^{3}$ times larger, the latter being estimated by making a quantum mechanical analogy of the Reynolds number minimum resistance criterion. This configuration greatly resembles the aether model of a frictionless fluid with ring vortices embedded in it proposed by Kelvin [3], except that the Planck aether is a quantum fluid composed of both positive and negative masses. As in Kelvin's model, the vortex lattice permits the propagation of transverse waves which for small amplitudes have exactly the same symmetry as those derived from Maxwell's equations. The vortex rings of the Planck aether are coupled through the longitudinal compression waves, which in the presence of the vortex lattice have a cut-off for a wave length larger than the vortex ring radius $r_{0}$. The transverse waves propagated through the vortex lattice have a lower wavelength cut-off at about the ring radius $r_{0}$. The value of $r_{0}$, estimated to be about $\sim 10^{3}$ times larger than the Planck length, fits well with the experimentally established grand unified energy, at which all the interactions transmitted by vector gauge bosons become equal, and which corresponds to a lower wavelength cut-off about $\sim 10^{3}$ times larger than the Planck length.

The correctness of the estimated value for the ring radius is supported by the interpretation of Dirac spinors as bound excitions made up from the positive and negative vortex resonance energy associated with an elliptic deformation of the vortex rings:

$$
\hbar \omega_{\mathrm{v}} \cong \pm m_{\mathrm{p}} c^{2}\left(r_{\mathrm{p}} / r_{\mathrm{o}}\right)^{2} .
$$

The compressional waves lead to the Newtonian gravitational potential by which the positive and negative mass of the vortex resonance are coupled. For masses of opposite sign, the energy of this interaction is positive, thereby providing the positive mass of the Dirac spinor. In terms of the Planck mass, the spinor mass $m$ turns out to be [1]

$$
m / m_{\mathrm{p}}=2\left(r_{\mathrm{p}} / r_{0}\right)^{6}=2\left(m_{\mathrm{G}} / m_{\mathrm{p}}\right)^{6} .
$$

For the typical value $m / m_{\mathrm{p}} \cong 2 \times 10^{22}$ (putting $m \cong 5$ $\mathrm{MeV}$ ), one obtains $r_{0} / r_{\mathrm{p}} \cong 5000$, in good agreement with the mass ratio of $m_{\mathrm{p}} / m_{\mathrm{G}}$, where $m_{\mathrm{G}}$ is the mass for the grand unified scale.

With the observed mass $m$ coming from the interaction energy of two very large and equal but opposite masses, our model can do without the Higgs field mechanism but, like the Higgs field equation, has in (1.5) the typical $\varphi^{3}$-term. With its natural in-built cut-off at the Planck length, our model eliminates all unphysical infinities. Unlike the divergent $\omega^{3}$ zero point energy spectrum of relativistic theories, our model has a cut-off at the Planck scale, resembling the Debye model of a solid.

\section{Vortex Substructure of the Planck Aether}

As previously shown [1], the proposed model is capable of reproducing the symmetries needed for quantum electrodynamics, but it is less obvious how it might lead to the observed quark-lepton symmetries of the standard model. An appealing feature of the Planck aether model is that it makes plausible the similarity between the phenomenology of high energy particle physics and solid state physics. In the model, all particles are quasiparticles of a substratum, like the phonons and excitions in a solid. The Higgs mechanism furthermore resembles the Landau-Ginzburg theory of superfluidity. Because of this broad similarity, one should expect that the quark-lepton symmetries should have a solid-state physics counterpart as well. We claim that such a resemblance is provided by the fractional quantum Hall effect. It occurs in a very pure thin sheet confining a two-dimensional electron gas. As Laughlin $[4,5]$ has shown, this electron gas can be described by the wave function

$$
\psi\left(\boldsymbol{r}_{1}, \boldsymbol{r}_{2}, \ldots, \boldsymbol{r}_{N}\right)=\left[\prod_{j<k}\left(z_{j}-z_{k}\right)^{\mu}\right]\left[\prod_{j} e^{-\left|z_{j}\right|^{2} /\left.4\right|^{2}}\right],
$$


where $z_{j}=x_{j}-i y_{j}$ is the coordinate of the $j$-th electron in complex notation and $l^{2}=\hbar c / e H$ (obtained from $m l^{2} \omega=\hbar$ and $\omega=e H / m c$ for the lowest Landau level). The magnetic field $H$ is directed perpendicular to the sheet. If $\mu$ is an odd integer, the wave function is completely antisymmetric, obeying Fermi statistics, and is made up from states of the first Landau level with the kinetic energy equal to $(1 / 2) \hbar \omega$ per electron. For the square of the wave function one has

$$
\begin{aligned}
|\psi|^{2} & =e^{-\beta H}, \\
\beta H & =2 \mu \sum_{j<k} \ln \left|\boldsymbol{r}_{j}-\boldsymbol{r}_{k}\right|+\left(1 / 2 l^{2}\right) \sum_{j}\left|\boldsymbol{r}_{j}\right|^{2},
\end{aligned}
$$

which is the probability distribution $|\psi|^{2}$ of a onecomponent two-dimensional plasma.

For $\mu=1$, the wave function is a Slater determinant, but this wave function does not describe the situation actually observed. Numerical calculations for four to six electrons done by Laughlin, rather, show that the wave function (2.1) for $\mu=3$ gives a much better agreement. It is this wave function which satisfactorily explains the fractional quantized Hall effect, in which plateaus in the conductivity are found to occur in multiple steps of $(1 / 3) e^{2} / h$.

The physical meaning of the wave function (2.1) can be understood if one keeps all electrons, except one, fixed in their position and carries out a closed loop motion of the one electron around a point at which the wave function vanishes. This displacement produces the phase shift

$$
\Delta \phi=(e / \hbar c) \oint \boldsymbol{A} \cdot \mathrm{d} \boldsymbol{s}=(e / \hbar c) \int \boldsymbol{H} \cdot \mathrm{d} \boldsymbol{f},
$$

where $\boldsymbol{H}=\operatorname{curl} \boldsymbol{A}$. Accordingly there should be

$$
Z=(e / \hbar c) \int \boldsymbol{H} \cdot \mathrm{d} \boldsymbol{f}
$$

vortices within the area $\int \mathrm{d} \boldsymbol{f}$. To satisfy the Pauli principle there must be at least one vortex at the position of each electron. In Laughlin's wave function there are exactly $\mu$ vortices for each electron. We therefore have to put $Z=\mu$. The fractional quantized Hall effect then simply means that the charge of one vortex is $e / 3$ provided $\mu=3$. In the two-dimensional electron fluid each electron, therefore, splits into three vortices of charge $e / 3$.

This result can be made plausible with Madelung's hydrodynamic formulation of quantum mechanics. The non-relativistic Schrödinger equation for one electron in a magnetic field is

$$
i \hbar \frac{\partial \psi}{\partial t}=\frac{1}{2 m}\left(-i \hbar \nabla-\frac{e}{c} A\right)^{2} \psi .
$$

With

$$
n=\psi^{*} \psi, \quad n \boldsymbol{v}=-\frac{i \hbar}{2 m}\left(\psi^{*} \nabla \psi-\psi \nabla \psi^{*}\right),
$$

the hydrodynamic form of (2.5) is given by

$$
\begin{aligned}
& \frac{\mathrm{d} \boldsymbol{v}}{\mathrm{d} t}=-\frac{1}{m} \operatorname{grad} Q+\frac{e}{m c} \boldsymbol{v} \times \boldsymbol{H}, \\
& \frac{\partial n}{\partial t}+\operatorname{div} n \boldsymbol{v}=0, \quad Q \equiv-\frac{\hbar^{2}}{2 m} \frac{\nabla^{2} \sqrt{n}}{\sqrt{n}},
\end{aligned}
$$

from which follows that $(v=0, \pm 1, \pm 2, \ldots)$

$$
\oint \boldsymbol{v} \cdot \mathrm{d} \boldsymbol{s}=\frac{h}{m} v-\frac{e}{m c} \int \boldsymbol{H} \cdot \mathrm{d} \boldsymbol{f} .
$$

It shows that the presence of a magnetic field causes the occurrence of the vortices in the electron fluid.

If a magnetic field is adiabatically applied to the electron fluid, the Helmholtz theorem

$$
\frac{\mathrm{d}}{\mathrm{d} t} \oint \boldsymbol{v} \cdot \mathrm{d} \boldsymbol{s}=0
$$

states that, if the circulation $\oint \boldsymbol{v} \cdot \mathrm{d} \boldsymbol{s}$ is zero before a magnetic field is applied, it remains zero thereafter. This, of course, does not imply that the circulation inside the contour taken in (2.9) can not differ from zero, because the circulation of different vortices can add up to zero, as it would be the case for four vortices with equal and opposite circulation. A vortex configuration with the total angular momentum $(1 / 2) \hbar$ could be constructed from two vortices with opposite circulation quantum number $v= \pm 1$, and one vortex with $v=1$ with the spin quantum numbers adding up to a total angular momentum $(1 / 2) \hbar$.

Variational calculations made by Laughlin show that the wave function is best described by a equilateral triangular vortex lattice, very much as in classical fluid dynamics, where in the Karman vortex street a triangular arrangement of the vortices has the greatest stability.

\section{The Quark-Lepton Symmetries}

The explanation of fractional charges in the fractional quantized Hall effect raises the question if the occurrence of fractional charges in quantum chromodynamics may have its cause in a vortex structure of the Planck aether. This would require for the aether to condense into thin sheets within which the vortices 
would be confined, but, in addition, there would have to be something acting like a magnetic field. Apart from these difficult dynamic questions, one can easily show that if the electron- and neutrino-wave functions can split up in vortices in a similar way as it happens in the fractionally quantized Hall effect, the quarklepton symmetries can be easily understood. The angular momentum of a vortex in units of $\hbar$ is equal to the circulation quantum number $v$. The vortices for which $v=1$ we call $\mathrm{A}$, those for which $v=0$ we call $\mathrm{B}$, and finally those for which $v=-1$ we all $\mathrm{C}$. The neutrino (v) and positron $\left(\mathrm{e}_{+}\right)$wave functions are then to be represented by six vortex states, with the lower indices giving the value for the electric charge of these vortex states:

$$
(v)=\left\{\begin{array}{l}
\mathrm{A}_{0} \\
\mathrm{~B}_{0}, \\
\mathrm{C}_{0}
\end{array} \quad\left(\mathrm{e}_{+}\right)=\left\{\begin{array}{l}
\mathrm{A}_{1 / 3} \\
\mathrm{~B}_{1 / 3} \\
\mathrm{C}_{1 / 3}
\end{array} .\right.\right.
$$

A second set of six vortex states is obtained by replacing the neutrino and positron with their antiparticles. That three vortices are needed is quite plausible because three vortices are needed at least to define a thin sheet, replacing the two-dimensional geometry in the Hall effect.

We claim that the first six vortex states can reproduce all the six $u$ and d quarks of the first family. How the neutrino and positron are composed of these vortex states is already shown in (3.1). With the indices $r, g$, $\mathrm{b}$ (red, green, blue) identifying what is called the color, we have for the three colors of the u quark:

$$
u_{r}=\left\{\begin{array}{l}
A_{1 / 3} \\
B_{1 / 3}, \\
C_{0}
\end{array} \quad u_{g}=\left\{\begin{array}{l}
A_{1 / 3} \\
B_{0} \\
C_{1 / 3}
\end{array}, \quad u_{b}=\left\{\begin{array}{l}
A_{0} \\
B_{1 / 3} . \\
C_{1 / 3}
\end{array}\right.\right.\right.
$$

For the three d quarks we have

$$
d_{r}=\left\{\begin{array}{l}
A_{0} \\
B_{0} \\
C_{-1 / 3}
\end{array}, \quad d_{g}=\left\{\begin{array}{l}
A_{0} \\
B_{-1 / 3}, \\
C_{0}
\end{array} \quad d_{b}=\left\{\begin{array}{l}
A_{-1 / 3} \\
B_{0} \\
C_{0}
\end{array} .\right.\right.\right.
$$

Because the vortices are substates of the leptons, color confinement then simply means that only those vortex configurations which can be combined into leptons are able to assume the form of free particles. Mesons are made up form quark-antiquark configurations, each containing three vortices and three antivortices.

If the vortices interact, they do this by the exchange bosons. Very much like a photon confined by a boundary, the bosons are here massive. And, very much like an electromagnetic wave in a wave guide, the waves assume a longitudinal component in addition to their transverse component. We claim that our model can thereby reproduce exactly the eight gluons of the standard model, communicating an interaction in between the vortices attached to the different quarks.

In our model the gluons are bosons transmitting angular momentum. To change an A vortex into a $\mathrm{B}$ vortex, a $\mathrm{B}$ vortex into a $\mathrm{C}$ vortex, or vice versa, requires a change in the angular momentum $\Delta L= \pm 1$, and to change an $\mathrm{A}$ vortex into a $\mathrm{C}$ vortex, or vice versa, a change by $\Delta L= \pm 2$ is needed. These changes can be made by just two angular momentum operators with $L=1$ and $L=2$, having $\sum(2 L+1)=2+1+4+1$ $=8$ states, equal to the number of gluons in $\mathrm{QCD}$. The transitions in QCD identified by red-green ( $\mathrm{r}-\mathrm{g})$, redblue (r-b), and green-blue (g-b), lead in our scheme to changes in the angular momentum of the vortices in the following way:

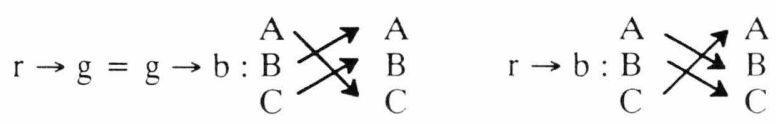

These transitions require four changes $\Delta L= \pm 1$ and two changes $\Delta L= \pm 2$, in total 6 changes. In addition there is the change for which $\Delta L=0$ :

$$
\mathrm{r} \rightarrow \mathrm{r}=\mathrm{g} \rightarrow \mathrm{g}=\mathrm{b} \rightarrow \mathrm{b}: \begin{aligned}
& \mathrm{A} \rightarrow \mathrm{A} \\
& \mathrm{B} \rightarrow \mathrm{B}, \\
& \mathrm{C} \rightarrow \mathrm{C}
\end{aligned},
$$

realized with the two $L_{z}=0$ components of the angular momentum operators for $L=1$ and $L=2$, which for this reason must be counted twice. Together with the 6 changes involving $\Delta L= \pm 1, \pm 2$ one has a total of 8 possible changes involving the exchange of angular momentum. The 8 gluons of QCD are, of course, not to be identified with these 8 angular momentum transmitting bosons but rather with certain combinations of them. A color-changing gluon would be always a superposition of a spin 1 and a spin 2 boson. A transition leaving the color unchanged would involve the superposition of spin 2 or spin 1 bosons. Our model, therefore, reduces color charge to nothing more than angular momentum. As we will see below, this assignment may help us to understand the physical origin of the charge phenomenon in general.

The same decomposition into vortex states done here for the first family can be repeated for the next higher families. And the weak interaction phenomenon is explained in our model by the exchange of bosons 
made up from spin 1 and spin 2 angular momentum transitions in between the vortices of the leptons given by (3.1).

We remark that our model can be compared with the rishon model, with the rishons turning out to be vortex states. The three hypercolor charges of the rishon model are the three angular momentum states $L=1,0,-1$ of the vortices. The prescription of the rishon model that only those configurations are possible which are color neutral with regard to the hypercolor, is explained in our model by the requirement that the vortex states must add up to zero angular momentum.

We note that collisions between polarized protons show a strong spin dependence of the interquark forces [6], which at least can be qualitatively understood by our vortex-model.

\section{The Origin of the Higgs Mechanism}

We have shown that the vortex substructure is rich enough to account for all the observed symmetries, but we now show that it may even explain the dynamic behavior. This is a much more difficult problem, but at least we can give some qualitative arguments pointing towards its solution.

As previously shown [1], the substratum model predicts very massive scalar quasiparticles of both mass signs, resulting from the resonances of the vortex rings, with the lowest resonance energy given by (1.9). Therefore we will now discuss the question of whether these quasiparticles might be identified with the Higgs bosons of the standard model. We note that, with a value of $r_{0} / r_{\mathrm{p}} \lesssim 10^{4}$, the resonance energy (1.9) would be $\gtrsim 10^{11} \mathrm{GeV}$, much too high to be detectable with any particle accelerator, including the SSC.

To explore this hypothesis in more detail, we write down the relativistic nonlinear wave equation derived from the Higgs-Lagrangian:

$$
\square \varphi+\varkappa^{2} \varphi-4 \lambda \varphi^{3}=0,
$$

where $\lambda>0$, and $x=m c / \hbar$, with $m$ equal to the mass of the Higgs meson. Because our model is exactly nonrelativistic, we have to make the transition of (4.1) to its nonrelativistic approximation. In the nonrelativistic limit we have to put

$$
-\frac{1}{c^{2}} \frac{\partial^{2} \varphi}{\partial t^{2}} \rightarrow \frac{2 i m}{\hbar} \frac{\partial \varphi}{\partial t}+\varkappa^{2} \varphi
$$

by which (4.1) becomes

$$
i \hbar \frac{\partial \varphi}{\partial t}=-\frac{\hbar^{2}}{2 m} \nabla^{2} \varphi-m c^{2} \varphi+\frac{2 \lambda \hbar^{2}}{m} \varphi^{3} .
$$

As in the original Higgs model, (4.3) has the static global solution [7]

$$
\varphi_{0}^{2}=\varkappa^{2} / 2 \lambda .
$$

Comparing (4.3) with the Hartree equation (1.5) for the mass $m_{\mathrm{p}}$, we may say that, according to our model, the Planck aether acts on $m_{\mathrm{p}}$ with a potential

$$
V=\left(f^{2} r_{\mathrm{p}}^{2} / m_{\mathrm{p}}\right)\left(\varphi^{2}-\varphi_{\mathrm{p}}^{2}\right)
$$

with the potential energy equal to $m_{\mathrm{p}} V$, and with $\varphi_{\mathrm{p}}^{2}=1 / 2 r_{\mathrm{p}}^{3}$. Assuming that the potential energy of a mass $m$ is likewise $m V$, we would have from (1.5) for a mass $m$ the nonlinear equation

$$
\begin{aligned}
i \hbar \frac{\partial \varphi}{\partial t} & =-\frac{\hbar^{2}}{2 m} \nabla^{2} \varphi+2 m c^{2} r_{\mathrm{p}}^{3}\left(\varphi^{2}-\varphi_{\mathrm{p}}^{2}\right) \varphi \\
& =-\frac{\hbar^{2}}{2 m} \nabla^{2} \varphi-m c^{2} \varphi+2 m c^{2} r_{\mathrm{p}}^{3} \varphi^{3} .
\end{aligned}
$$

A comparison of (4.6) with (4.3) gives a value for the coefficient of the quartic in the Higgs Lagrangian. Putting $r_{0} / r_{\mathrm{p}} \cong 5 \times 10^{3}$, one finds

$$
\lambda=\varkappa^{2} r_{\mathrm{p}}^{3}=r_{\mathrm{p}}\left(r_{\mathrm{p}} / r_{0}\right)^{4} \cong 2.5 \times 10^{-48} \mathrm{~cm} .
$$

In the standard model the weak vector boson mass can be expressed by the "weak magnetic vector potential" $\boldsymbol{A}$ of the WSG theory as follows (with the vacuum gauge $\boldsymbol{A}=0$ ):

$$
m_{\mathrm{w}} c^{2}=e A,
$$

but also through the universal Fermi constant $G_{\mathrm{F}}$ by

$$
m_{\mathrm{w}} c^{2}=2^{1 / 4} \mathrm{~g} \sqrt{\hbar c / G_{\mathrm{F}}} \cong 85 \mathrm{GeV},
$$

where $g$ is the semiweak coupling constant. Since in our substratum model a lepton might split into three vortices, it is suggested that these vortices are line vortices in a sheet of thickness $\delta$, with the vortices ending at the two surfaces of the sheet. If this is so, then the "weak magnetic field" in the sheet must be $H \sim A / \delta$. And because of $m_{\mathrm{w}} c \delta \cong \hbar$, we find from (4.8) an expression for $H$ :

$$
H=\left(m_{\mathrm{w}} c^{2}\right)^{2} / e \hbar c \cong 10^{26}[\mathrm{esu}],
$$

which corresponds to a huge weak magnetic field of $\sim 10^{26}$ Gauss. Like a magnetic field it produces a Lorentz-force. It can be shown to be strong enough to 
produce a vortex structure, as it is known from the fractional quantized Hall effect. According to (2.8) the magnetic field needed must be at least of the order

$$
H \sim \hbar c / e r^{2},
$$

where $r$ is the Larmor radius. For relativistic velocities $r$ is of the order

$$
r \sim m_{\mathrm{w}} c^{2} / e H
$$

Eliminating $r$ from (4.11) and (4.12) gives the same value of $H$ as (4.10). Furthermore, from

$$
H \sim 4 \pi n e \delta
$$

we find for the number density of charges in the sheet

$$
n=\cong\left(m_{\mathrm{w}} c / \hbar\right)^{3} \cong 10^{51} \mathrm{~cm}^{-3}
$$

(provided each intermediate vector boson has the unit charge $e \sim \sqrt{\hbar c}$ ).

If the energy density of the Higgs masses are of the same order of magnitude as the energy density of the weak vector bosons, one has for the number density of the Higgs mesons

$$
n_{\mathrm{H}} / n \cong m_{\mathrm{w}} / m \cong 10^{-9}, \quad n_{\mathrm{H}} \sim 10^{42} \mathrm{~cm}^{-3} .
$$

Because $n_{\mathrm{H}}$ is still very small compared to the number density $r_{\mathrm{p}}^{-3} \sim 10^{99} \mathrm{~cm}^{-3}$ of the Planck masses, a very small increase in the substratum density caused by a local condensation into thin sheets would be sufficient to produce the vortices.

A more interesting question is if the mass of the intermediate vector boson can somehow be derived. In the standard model $\pi$-mesons are bound states made up from quarks and their antiquarks. It is, therefore, plausible to assume that the intermediate vector bosons are bound states made up from vortices and their respective antivortices, into which the leptons can split according to our model. The mass formula (1.10) for a Dirac spinor was derived from the equation

$$
m=2 G\left|m_{\mathrm{v}}\right|^{3} / \hbar c=\left|m_{\mathrm{v}}\right|^{3} / m_{\mathrm{p}}^{2},
$$

where $\left|m_{\mathrm{v}}\right|=m_{\mathrm{p}}\left(r_{\mathrm{p}} / r_{0}\right)^{2}$. In (4.16) $m$ is the mass resulting from the interaction of two quasi-particles of opposite mass $\pm m_{\mathrm{v}}$. We may likewise apply the same formula to the interaction of two vortices of opposite mass formed from the fluid of quasiparticles of mass $\pm m_{\mathrm{v}}$. The effective mass of a vortex should be determined by its kinetic energy. For a vortex all masses attached to the vortex and having a nonzero kinetic energy can contribute to the zero point fluctuations.
For the effective vortex mass $m_{\mathrm{v}}^{*}$, we therefore have

$$
m_{\mathrm{v}}^{*} c^{2}=\frac{1}{\pi r_{\mathrm{p}}^{2}} \int_{r_{\mathrm{p}}}^{r}\left(m_{\mathrm{v}} / 2\right) v^{2} 2 \pi r \mathrm{~d} r .
$$

With the vortex velocity given by

$$
v=\mathrm{c}\left(r_{\mathrm{p}} / r\right)
$$

we find that

$$
m_{\mathrm{v}}^{*}=m_{\mathrm{v}} \ln \left(r / r_{\mathrm{p}}\right) .
$$

Assuming that the average distance between the vortices is by order of magnitude $r \cong \hbar / m_{\mathrm{w}} c$, we find by inserting into (4.16) after replacing $m_{\mathrm{v}}$ by $m_{\mathrm{v}}^{*}$ and setting $m \cong=m_{\mathrm{w}} / 2$, since the intermediate vector boson shall be composed to a vortex and its antivortex of equal mass, that

$$
m_{\mathrm{w}} \cong 4\left(m_{\mathrm{v}}^{3} / m_{\mathrm{p}}^{2}\right) \ln ^{3}\left(m_{\mathrm{p}} / m_{\mathrm{w}}\right) .
$$

With $m / m_{\mathrm{p}}=2\left(m_{\mathrm{v}} / m_{\mathrm{p}}\right)^{3}$ we can write for $(4.20)$

$$
\frac{m_{\mathrm{w}}}{m}=2 \ln ^{3}\left(\frac{m_{\mathrm{p}}}{m} \frac{m}{m_{\mathrm{w}}}\right) .
$$

With the empirical value of the electron-Planck-mass ratio $m_{\mathrm{p}} / m \cong 2.4 \times 10^{22}$, we find that $m_{\mathrm{w}} / m \cong 1.2 \times 10^{5}$ and, therefore, $m_{\mathrm{w}} \cong 60 \mathrm{GeV}$, not too far off the correct value $m_{\mathrm{w}} \cong 85 \mathrm{GeV}$. A better agreement can hardly be expected in view of the crude estimate made.

\section{The Origin of Charge}

A fundamental problem in elementary particle physics is the origin of charge. It enters into the equations as an empirical coupling constant, but otherwise remains completely unexplained. However, it was shown [1] that in the Planck aether hypothesis the gravitational charge at least has a very plausible explanation. It results there from the zero point fluctuations of the Planck masses bound to the core of the positive and negative mass ring vortices formed in the Planck aether.

According to the uncertainty principle, the kinetic energy density of the fluctuating Planck masses positioned in the vortex core within a volume of radius $2 r_{\mathrm{p}}$ is

$$
\varepsilon \cong \hbar c / 8 \pi r_{\mathrm{p}}^{4} .
$$

These fluctuations are the source of virtual compression waves propagating with the velocity of light, and they produce an attractive inverse square law force $F$ 
with an energy density $F^{2} / 8 \pi$. For positive Planck masses this energy density is actually negative, but so is there the kinetic energy density (5.1), because it is the kinetic energy density of a hollow vortex filament. For vortices formed from negative Planck masses the situation is reversed. Equating for $r=r_{\mathrm{p}}$ the energy density $F^{2} / 8 \pi$ with (5.1) and solving for $F$ one finds that

$$
F=-\frac{\sqrt{G} m_{\mathrm{p}}}{r^{2}}
$$

which is just the attractive Newtonian force exerted by one Planck mass. For Dirac spinors of mass $m \ll m_{\mathrm{p}}$ the gravitational charge is reduced to $\sqrt{G} m$ because $m$ is there the residual mass resulting from the gravitational interation energy of two large positive and negative masses of the Planck aether of which the Dirac spinor is composed *.

At the grand unified energy, which in our model is the scale of the ring radius of the positive and negative mass ring vortices, all the interactions become equal with the exception of the gravitational interaction. At the Planck scale the gravitational interaction $G m_{\mathrm{p}}^{2} /$ $\hbar c=1$, but at the grand unified scale the dimensionless constants of the strong, weak and electromagnetic interaction are smaller. The fact that these interaction constants are not many orders of magnitude smaller than $G m_{\mathrm{p}}^{2}$ suggests that their origin as well is located in the zero point fluctuations, with the reduction in the interaction constants less than $G m_{\mathrm{p}}^{2}$ resulting from polarization effects. This hypothesis still must explain 1) why the electromagnetic interaction leads to a repulsion, 2) why the charges are equal at the grand unified scale, 3 ) why the weak interaction is coupled to an axial current vector, and 4) why some interactions enter in non-Abelian gauge theories.

In the Planck aether hypothesis the fundamental ring vortices are coupled with each other through virtual compression waves in the wavelength region $r_{\mathrm{p}}<\lambda<r_{0}$. This coupling leads to a mechanical wave which for small amplitudes has the same form as the transverse electromagnetic wave derived from Maxwell's equations. Because this wave is described by a vector field equation, it must lead to a repulsion. At the scale of the ring radius $r_{0}$, the energy density of the

* In the Planck aether model all fields are replaced by mechanical motions of the Planck masses, and a gravitational mass $m \ll m_{\mathrm{p}}$ can there arise from the uncompensated kinetic fluid energy of the positive and negative masses of the substratum. field transmitted by these waves is

$$
E^{2} / 8 \pi \cong e^{2} / 8 \pi r_{0}^{4},
$$

where $e$ is the electromagnetic coupling constant. To determine its value resulting from the zero point fluctuations, we note that at the scale $r_{0}$ we have

$$
\gamma m_{\mathrm{v}} r_{0} c \cong \hbar,
$$

where $m_{\mathrm{v}}=m_{\mathrm{p}}\left(r_{\mathrm{p}} / r_{0}\right)^{2}$, and where $\gamma=\left(1-v^{2} / c^{2}\right)^{-1 / 2}$ $\cong r_{0} / r_{\mathrm{p}}$ with $v$ the azimuthal velocity of the rapidly circulating pole-dipole particle producing the Dirac spinor [1]. From (5.4) we have

$$
\gamma m_{\mathrm{v}} \cong\left(r_{\mathrm{p}} / r_{0}\right) m_{\mathrm{p}},
$$

leading within the volume $(4 \pi / 3) r_{0}^{3}$ to the zero point energy density

$$
\varepsilon \cong(1 / 6) \gamma m_{\mathrm{v}} c^{2} /(4 \pi / 3) r_{0}^{3} \cong \hbar c / 8 \pi r_{0}^{4} .
$$

Equating (5.3) with (5.6) yields for the value of the electric charge at the ring scale

$$
e^{2} \cong \hbar c \text {. }
$$

By extrapolating the coupling constants to very high energies one finds $e^{2} / \hbar c \sim 1 / 40$ instead. A value $e^{2} / \hbar c$ $\sim 1 / 40$ would mean that at the grand unified scale the Planck aether has a dielectric constant $\varepsilon \sim \sqrt{40} \sim 6$, resulting from polarization effects not treated in our crude estimate.

The axial current interaction of the standard model occurs only for configurations involving in our model vortex substructures, in which the leptons split up in a triplet of vortices. Very much like a bound particle can execute radial zero point oscillations, a vortex can execute azimuthal zero point oscillations. With the moment of inertia $\Theta \cong m_{\mathrm{p}} r_{\mathrm{p}}^{2}$ and angular velocity $\omega$ the magnitude of these zero point fluctuations is determined by the uncertainty principle for rotational motion

$$
\Theta \omega \cong \hbar,
$$

leading to an energy density by order of magnitude equal to the energy density of the radial oscillations. The vortices thereby become the source of virtual vector field waves which by order of magnitude again must have the coupling strength $g^{2} \sim \hbar c$. However, because the virtual rotational fluctuations have the character of an axial vector, the current to which these interactions couple must be an axial vector as well.

The origin on non-Abelian gauge theories can be explained as a result of vortex-vortex interaction 
through their radial and rotational zero point fluctuations. These vortices behave like small electrically charged magnets, and the total vortex-vortex interaction must, therefore, be a superposition of electric- and magnetic-type interactions. The static electric force exerted by magnet 1 on magnet 2 is

$$
F_{\mathrm{e}}=-e \nabla \Phi_{1},
$$

where $\Phi_{1}$ is the scalar electric potential produced by the electric charge on magnet 1 . To compute the static magnetic force, we consider two magnets of moments $\boldsymbol{m}_{1}, \boldsymbol{m}_{2}$, separated by the distance $r$, with the magnetic fields produced by them expressed through their vector potentials.

$$
\boldsymbol{A}_{1}=\frac{\boldsymbol{m}_{1} \times \boldsymbol{e}_{\mathrm{r}}}{r^{2}}, \quad \boldsymbol{A}_{2}=-\frac{\boldsymbol{m}_{2} \times \boldsymbol{e}_{\mathrm{r}}}{r^{2}},
$$

where it is assumed the $\boldsymbol{e}_{\mathrm{r}}$ is $\perp$ to $\boldsymbol{m}_{1}$ and $\boldsymbol{m}_{2}$, and where $e_{\mathrm{r}}$ is a unit vector along $r$. The magnetic force on $\boldsymbol{m}_{2}$ by $\boldsymbol{m}_{1}$ is then given by

$$
F_{\mathrm{m}}=\nabla\left(\boldsymbol{m}_{2} \cdot \operatorname{curl} \boldsymbol{A}_{1}\right)=6 \boldsymbol{A}_{1} \cdot \boldsymbol{A}_{2} \boldsymbol{e}_{\mathrm{r}} .
$$

For three interacting vortices, three vector potentials are likewise needed. The nonlinear quadratic term in (5.11) is typical for terms occurring in Yang-Mills theories. The expressions for $F_{\mathrm{e}}$ and $F_{\mathrm{m}}$ are valid for static fields. For nonstatic fields the forces are obtained by their relativistic generalizations, because in the dynamic substratum interpretation of special relativity only those configurations are stable whose lifetime is large compared with the Planck time [1]. The only possible generalizations satisfying this dynamic selection principle are just the relativistically invariant Yang-Mills type force fields:

$$
F_{\mu \nu}^{k}=\partial_{\mu} A_{v}^{k}-\partial_{v} A_{\mu}^{k}-g^{-1} f_{l m}{ }^{k} A_{\mu}^{l} A_{v}^{m},
$$

where $f_{l m}{ }^{k}$ are the dimensionless structure constants and where $g$ is a coupling constant having the dimension of an electric charge. In the SU2 electroweak model one has $k=1,2,3$ and $f_{l m}{ }^{k}=\varepsilon_{l m}{ }^{k}$, where $\varepsilon_{l m}{ }^{k}$ is the Levi-Civita completely antisymmetric tensor. In Yang-Mills theories of the weak and strong interactions $g$ must be inserted "by hand". In our model its value can be determined from the zero point fluctuations.

In the Planck aether there is an equal number of positive and negative Planck masses, and because all charges have their origin in the zero point fluctuations of the Planck masses, the equal number of positive and negative Planck masses then explains why the
Planck Masses

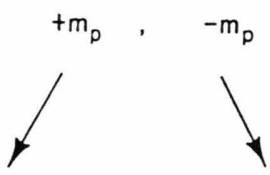

Vortex Rings

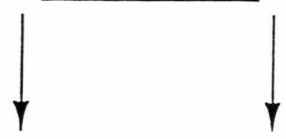

Vortex Ring Resonance $\quad\left(r_{0}{ }^{2} / r_{p}\right)$

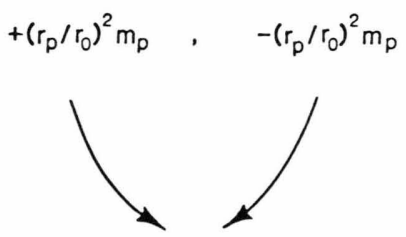

Lepton Dirac Spinors $\left(r_{0}^{6} / r_{p}^{5}\right)$

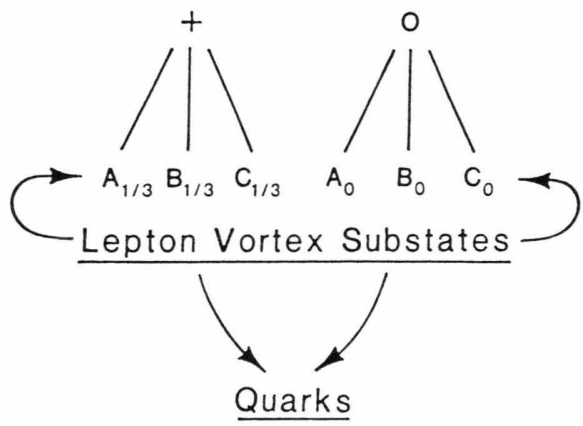

Fig. 1. Different structures and scales in the Planck aether hypothesis.

sum of all charges is zero. This is true for the electric charges, but it is also true for the sum of all color charges, always adding up to a colorless combination. For the sum of the gravitational charges it is true if the negative gravitational potential energy cancels the positive energy of all gravitationally interacting masses. Since in our model positive masses result from the positive gravitational energy of positive masses interacting with negative masses, it simply means that if without the expenditure of energy positive masses are formed out of the Planck aether, the positive gravitational energy set up between equal masses of opposite sign must be exactly cancelled by the negative gravitational energy set up in between the positive masses, with this positive mass, resulting from the interaction 


\section{Meson-States}

Lepton-Antilepton $=$ Positronium...

higher families

Quark-Antiquark $=\pi^{+}$Meson..., higher families

Vortex-Antivortex $=W, Z$ Bosons..., higher families

Fig. 2. The different meson states.

of the positive with the negative masses. Astronomical data, in fact, suggest that the positive energy of the universe is balanced by its negative gravitational potential energy, and which is another way of saying that the cosmological constant is zero. The vanishing of the cosmological constant, and the other empirical fact according to which the sum of all other charges is zero, has in our model, therefore, the same cause, which is the equal number of positive and negative Planck masses in the Planck aether.

\section{The Higher Families}

In the Planck aether model the second and third families can be explained as radial internal excitations of pole-dipole configurations. The value of the first excited state corresponding to a radial pulsation has been computed by Bopp [8], with the result that it is about $\sim 200$ times larger than the ground state energy, a result in good agreement with the experimentally obtained muon-electron mass ratio. The whole reasoning we had outlined for the first family, then simply repeats itself for the higher families. In the

[1] F. Winterberg, Z. Naturforsch. 43a, 1131 (1988).

[2] E. Madelung, Z. Physik 40, 322 (1926).

[3] W. Thomson (Lord Kelvin), Phil. Mag. 24, 342 (1887).

[4] R. B. Laughlin, Phys. Rev. 27 B, 3383 (1983).

[5] R. B. Laughlin, Phys. Rev. Lett. 50, 1395 (1983).

[6] A. D. Krisch, Proc. 4th Int. Symp. Polarization Phenomena in Nuclear Reactions, Birkhäuser, Basel 1976. - P. R. Cameron et al., Phys. Rev. D 32, 3070 (1985). second family it is the muon and mu-neutrino which would split up into sub-vortex states, with combinations of them producing the strange and charmed quarks.

Because the intermediate vector bosons of the WSG theory are in our model composed of a vortex-antivortex pair from the first generation, there should be two more, much heavier intermediate vector bosons made up from vortices of the two next higher generations. Assuming that the mass ratios of the vector bosons are about equal to the mass ratios of the leptons, the next higher vector boson should, therefore, have a mass of $\sim 2 \times 10^{4} \mathrm{GeV}$.

In Fig. 1 we have displayed the different structures and scales as they are conjectured in the Planck aether hypothesis. In Fig. 2, the different meson-states are displayed. Because all these mesons are derived from the leptons, they must include positronium as such a state.

Compared with the rishon model, the rishons are in our model replaced by the $A, B$, and $C$ vortices. Our model, however, does not share the two fundamental problems of the rishon model, which are 1) the energy mismatch for the composed states, and 2) its inability to explain the higher families as a result of internal radial excitations of the rishons. The reason why our model does not have these problems is that it explains Dirac spinors as objects composed from two large masses of opposite sign, with the residual mass coming from the positive gravitational energy. Even though the rishon model can account for all observed fermions, it fails to give an explanation for the origin of the vector gauge bosons, explained in our model by collective substratum excitations.

[7] J. Iliopoulos, Contemp. Phys. 21, 159 (1980).

[8] F. Bopp, Z. Physik 125, 615 (1949).

[9] A. D. Sakharov, Sov. Phys. Doklady 12, 1040 (1968). 